\title{
Research on Application of Project Management Maturity Model in Risk Management of Electrical Engineering
}

\author{
JiaNan Shan ${ }^{1, a}$, RenXiang Lu ${ }^{1}$ \\ ${ }^{1}$ Shanghai DianJi University, Business Department, Shanghai, China
}

\begin{abstract}
Based on the analysis of foreign classic maturity models and the actual situation of electrical engineering projects, this paper constructs a suitable three-dimensional project risk management maturity model and establishes a project risk management evaluation index system. Through fuzzy comprehensive evaluation method and analytic hierarchy process, the key element indicators are evaluated and weighted, and the maturity score is obtained, which finally reflects the maturity of project risk management. Finally, through empirical analysis, we found out the shortcomings of enterprise project risk management and proposed the direction for improvement.
\end{abstract}

\section{The theory and development status of project management maturity model}

The Project Management Maturity Model (PMMM) is a standard to help organizations measure project management capabilities, and it is also a framework method for evaluating and improving project management. It depicts the process by which a project system develops over time and its project management capabilities reach the highest level and also reflects the ability to reliably achieve specific engineering goals in the face of unknown risks $^{[1]}$.

The research on the maturity model first started in 1987. The software capability maturity model integrated CMMI was developed by the Software Institute (SEI) of Carnegie Mellon University in the United States ${ }^{[2]}$. With the continuous expansion of the scope of application and the continuous updating of the model itself, the CMMI model has become a comprehensive model with higher authority and has pointed out the direction for the construction of subsequent models. Domestic related research started relatively late. In the field of engineering, Jiancheng $\mathrm{Su}^{[3]}$ conducted risk analysis for Kangbashi distribution network projects and proposed corresponding maturity models; Wei Chen ${ }^{[1]}$ optimized the enterprise project management plan based on the P3M3 model; Zhanqiao Qin ${ }^{[4]}$ used the gray comprehensive evaluation model for empirical research which proved the feasibility of her method in the field of project management; Ke Chen $^{[5]}$ used the OPM3 model to conduct application evaluation research on domestic engineering companies and found the problems in the application process and proposed related solutions; $\mathrm{Yi} \mathrm{Lu}$ and $\mathrm{Han} \mathrm{Li}^{[6]}$ took structural equation principle and AMOS7.0 as the evaluation method of project management maturity. The problem that the management variables are difficult to be accurately and directly measured in the project management maturity evaluation is solved by them.

\section{Construction of project risk management maturity model}

\subsection{The structure of the project risk management maturity model}

Based on the characteristics of electrical engineering projects, this paper builds a three-dimensional model of electrical engineering project risk management maturity based on the existing models. The first dimension is the key indicator elements of project risk management; the second dimension is the project risk management process; and the third dimension is the project risk management maturity level ${ }^{[7]}$. The three-dimensional structure is shown in Figure1.

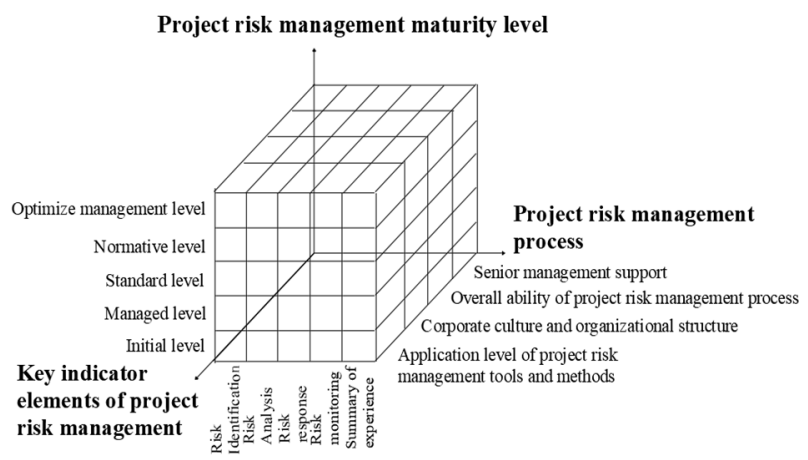

Fig1. Three-dimensional structure model of project risk management maturity 


\subsection{Project risk management maturity level classification}

By referring to the classification of the classic project risk management maturity model, this paper is based on the CMMI model and combined with the actual process of the electrical engineering project to divide the electrical engineering project risk management maturity model into five levels: initial level, Management level, standard level, normative level, optimized management level. The classification of project management maturity level is shown in Figure 2.

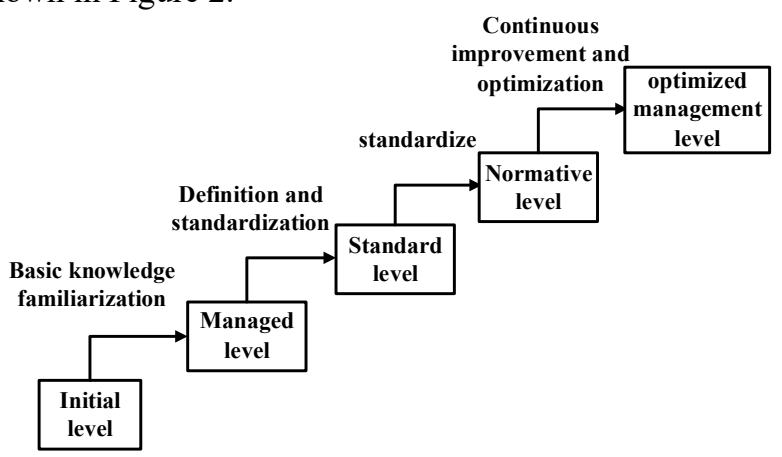

Fig2. Project risk management maturity level classification

\subsubsection{Level 1: Initial level}

At this level, enterprises lack sufficient risk management capabilities, their risk management awareness is relatively weak. Risk management systems are relatively vague and management measures are relatively disorderly. The relevant project management personnel of the enterprise need further training to avoid relying only on previous engineering experience and maintaining the basic operation of the project only.

\subsubsection{Level 2: Managed level}

Under the second level, enterprises have formulated a basic risk management system. Relevant personnel have a preliminary understanding of risk management knowledge and begin to form a fixed risk management team. They will formulate response plans and take certain measures when facing with project risks. However, due to insufficient management capabilities, there will be some problems in the planning process and implementation.

\subsubsection{Level 3: Standard level}

Enterprises at this level already have a set of established risk management rules and regulations, and they can strictly abide by them. The cooperation between various departments is also more proficient, and common project management methods and tools can be used for risk identification and risk monitoring, which can basically ensure the realization of project quality and schedule goals, and project risk management capabilities are basically mature.

\subsubsection{Level 4: Specification level}

At this level, the enterprise project management team can formulate detailed plans and strictly implement the quality, schedule, and cost goals of project management. The enterprise's risk response ability is very mature, they can use project management software to accurately analyze and control the realization of project goals and establish a risk database to provide reference for subsequent risk management.

\subsubsection{Level 5: Optimized management level}

Enterprises at this level are able to use project management tools and methods proficiently. Corporate managers are already very familiar with project risk management theories and are able to innovate based on actual conditions. The company's project risk management capabilities are already at the highest level, and the management system can be continuously optimized from the perspective of developments trategy.

\section{The establishment of evaluation index system for project risk management maturity}

\subsection{Steps to establishing evaluation index system}

Project management maturity is affected by many factors. Due to the long-term nature and complexity of electrical engineering projects, the evaluation index system of electrical engineering project management maturity is relatively large. This paper has formulated five steps for the establishment of the risk management maturity evaluation index system of electrical engineering projects: 1. Summarizing the factors affecting the risk management maturity of electrical engineering projects; 2 . Conducting questionnaire surveys and statistics on relevant personnel; 3. Determining project risks Management maturity evaluation index; 4. Determining the corresponding weight of each evaluation index; 5. Establishing a maturity evaluation index system.

Based on the current status of electrical engineering enterprise management, this paper sets up four first-level indicators of the project risk management maturity evaluation system, including the support of project senior managers, the overall level of the project management process, corporate culture and organizational structure, and project management tools and methods. Under the four first-level indicators, several second-level indicators need to be selected. In this paper, the project managers and related management personnel of some enterprises were interviewed and surveyed through the questionnaire survey method. Finally 155 valid questionnaires were obtained. This paper uses Cronbach's alpha test method to analyze the reliability and validity of the questionnaire data results, compares and analyzes the importance of multiple indicators, and finally obtains 15 secondary 
indicators. Project risk management evaluation index system is shown in Figure 3.

\subsection{Evaluation index system scoring standard}

This paper uses the fuzzy comprehensive evaluation method to comprehensively evaluate various indicators of project risk management maturity. The fuzzy comprehensive evaluation process is as follows:

(1) Since this paper divides the maturity level into five levels, it is scored according to the five levels for 15 secondary indicators ${ }^{[8]}$. First, assign values to the five levels from low to high, namely 1, 2, 3, 4, and 5. Then determine the comment level domain, and formula (1) can be obtained from the assignment.

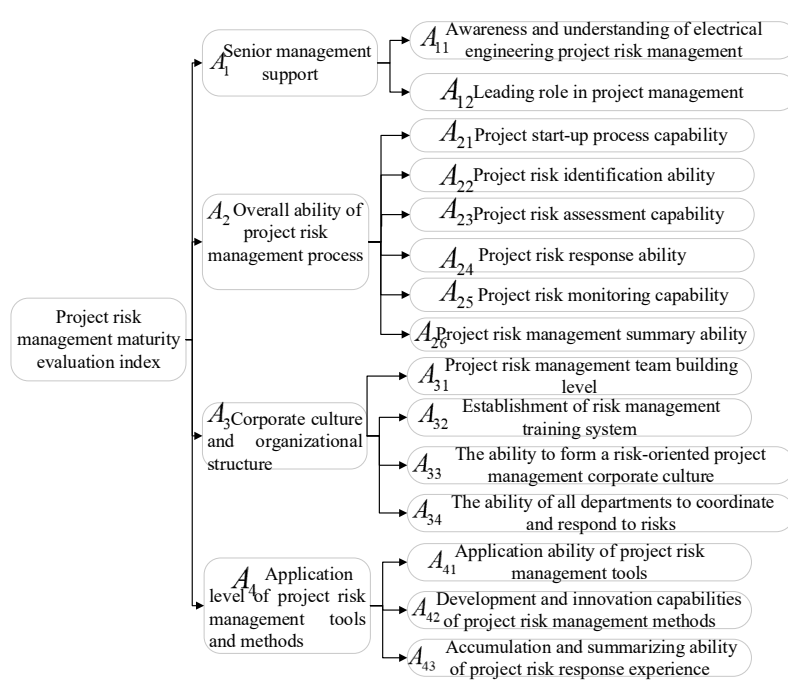

Fig3. Project risk management evaluation index system

(2) The corresponding comment level of each index at each maturity level is shown in Table 1.

Table 1. Scoring standards for various indicators

\begin{tabular}{c|c|c|c|c|c|c}
\hline \multirow{2}{*}{$\begin{array}{c}\text { First level } \\
\text { indicator }\end{array}$} & $\begin{array}{c}\text { Second level } \\
\text { indicator }\end{array}$ & \multicolumn{5}{|c}{ Project management maturity level } \\
\cline { 3 - 7 }$A_{1}$ & $A_{11}$ & bad & poor & normal & good & better \\
\cline { 2 - 7 } & $A_{12}$ & very few & little & general & many & more \\
\hline \multirow{4}{*}{$A_{2}$} & $A_{21}$ & very low & low & general & high & very high \\
\cline { 2 - 7 } & $A_{22}$ & very low & low & general & high & very high \\
\cline { 2 - 7 } & $A_{23}$ & very low & low & general & high & very high \\
\cline { 2 - 7 } & $A_{24}$ & very low & low & general & high & very high \\
\cline { 2 - 7 } & $A_{25}$ & very low & low & general & high & very high \\
\hline \multirow{5}{*}{$A_{36}$} & very low & low & general & high & very high \\
\cline { 2 - 7 } & $A_{31}$ & very low & low & general & high & very high \\
\cline { 2 - 7 } & $A_{32}$ & unsound & lack & normal & regular & perfect \\
\cline { 2 - 7 } & $A_{33}$ & very low & low & general & high & very high \\
\cline { 2 - 7 } & $A_{34}$ & very low & low & general & high & very high \\
\hline$A_{41}$ & poor & rusty & normal & familiar & proficient \\
\hline \multirow{4}{*}{$A_{4}$} & $A_{42}$ & very low & low & general & high & very high \\
\cline { 2 - 7 } & $A_{43}$ & very low & low & general & high & very high \\
\hline & & & &
\end{tabular}

(3) According to the scores of various indicators shown in Table 3, a fuzzy relationship matrix is established, as shown in formula (2).

$$
R_{i}=\left(\begin{array}{cccc}
r_{11} & r_{12} & \ldots & r_{1 m} \\
r_{21} & r_{22} & \ldots & r_{2 m} \\
\ldots & \ldots & \ldots & \ldots \\
r_{j 1} & r_{j 2} & \ldots & r_{j m}
\end{array}\right)
$$

Among them, $m$ is the number of scoring standards corresponding to each maturity level, and $j$ is the number of secondary indicators corresponding to a certain maturity level.

\subsection{Determination of evaluation index weight}

This paper uses the analytic hierarchy process to determine the weight of the evaluation index. First, select 10 project managers in the electrical department of the enterprise and score each evaluation index according to the degree of importance. Then obtain the evaluation 
matrix of each index according to the score result of each index, calculate the maximum characteristic root $\lambda_{\max }$ and weight of each index corresponding matrix $W_{i}$, then use the analytic hierarchy process software yaahp to test the consistency of the evaluation matrix, the test formula is as formula (3) shown ${ }^{[9]}$.

$$
C I=\frac{\lambda_{\max }-n}{n-1}
$$

It is generally believed that when the consistency test index $C I<0.1$, the consistency of the evaluation matrix is better. So the weight of the corresponding evaluation index is larger, indicating that the index is relatively important. On the contrary, the larger the value, the worse the consistency of the evaluation matrix, the smaller the weight of the corresponding evaluation index, and the worse the reliability of the index. Through calculation, the analysis results of the first-level indicators are shown in Table 2.

Table 2. $\lambda_{\max }, C I$ and $W_{i}$ of the first-level index

\begin{tabular}{cccc}
\hline First level indicators & $\lambda_{\max }$ & $C I$ & $W_{i}$ \\
\hline Senior management support & 2.0000 & 0.0000 & 0.2359 \\
\hline $\begin{array}{l}\text { Overall ability of project } \\
\text { risk management process }\end{array}$ & 6.5396 & 0.0856 & 0.5426 \\
\hline $\begin{array}{l}\text { Corporate culture and } \\
\text { organizational structure }\end{array}$ & 4.1179 & 0.0442 & 0.1221 \\
\hline $\begin{array}{c}\text { Application level of project } \\
\text { risk management tools and methods }\end{array}$ & 3.0536 & 0.0516 & 0.0995 \\
\hline
\end{tabular}

It can be seen from Table 2 that the consistency ratio of the first-level indicators is all less than 0.1 . It means that the consistency test is passed. Then, the weight of each secondary index can be obtained by calculation, as shown in Table 3 .

Table 3. The weight of second-level indicator

\begin{tabular}{ccccccccc}
\hline$A$ & $A_{11}$ & $A_{12}$ & $A_{21}$ & $A_{22}$ & $A_{23}$ & $A_{24}$ & $A_{25}$ & $A_{26}$ \\
\hline$W_{i}$ & 0.6667 & 0.3333 & 0.0729 & 0.1342 & 0.1647 & 0.4334 & 0.1119 & 0.0829 \\
\hline$A$ & $A_{31}$ & $A_{32}$ & $A_{33}$ & $A_{34}$ & $A_{41}$ & $A_{42}$ & $A_{43}$ & \\
\hline$W_{i}$ & 0.3908 & 0.3048 & 0.1460 & 0.1584 & 0.4126 & 0.2599 & 0.3275 & \\
\hline
\end{tabular}

\subsection{Calculation of evaluation result}

Multiply the secondary index weight matrix $W_{i}$ and the secondary index evaluation matrix $R_{i}$ to obtain the final evaluation result matrix of each index, as shown in formula (4).

$$
\begin{aligned}
& B_{i}=W_{i} \circ R_{i} \\
& =\left[\begin{array}{llll}
W_{i 1} & W_{i 2} & \ldots & W_{i j}
\end{array}\right] \circ\left(\begin{array}{cccc}
r_{11} & r_{12} & \ldots & r_{1 m} \\
r_{21} & r_{22} & \ldots & r_{2 m} \\
\ldots & \ldots & \ldots & \ldots \\
r_{j 1} & r_{j 2} & \ldots & r_{j m}
\end{array}\right)
\end{aligned}
$$

Multiplying the evaluation result matrix $B_{i}$ by the comment rating matrix $V$ can obtain the scores of various key indicators and maturity levels, as shown in formula
(5). Then multiply the weight matrix of the first-level index $W$ by the final evaluation result matrix $B_{i}$ of each index, then the overall final score of the risk management maturity of the electrical engineering project, as shown in formula (6).

$$
\begin{gathered}
F_{i}=B_{i} \times V^{T} \\
A=W \times B_{i}
\end{gathered}
$$$$
=\left[\begin{array}{llll}
W_{1} & W_{2} & W_{3} & W_{4}
\end{array}\right] \times\left(\begin{array}{c}
B_{1} \\
B_{2} \\
\cdots \\
B_{i}
\end{array}\right)
$$

$$
F=A \times V^{T}
$$

Then multiply the overall final score and the comment level matrix to get the final project risk management 
maturity level, as shown in formula (7). The corresponding maturity level of the scores of each key indicator is shown in Table 4.

Table 4. Final score maturity level comparison

\begin{tabular}{c|c}
\hline Final score & Maturity level \\
\hline$[1,2)$ & Initial level \\
\hline$[2,3)$ & Managed level \\
\hline$[3,4)$ & Standard level \\
\hline$[4,5)$ & Normative level \\
\hline 5 & Optimized management level \\
\hline
\end{tabular}

\section{Case Analysis}

In view of the above analysis methods, this paper takes a private company $\mathrm{H}$ in Shanghai as an example, and verifies the feasibility of the analysis method in this paper by evaluating the risk management maturity of $\mathrm{H}$ company's electrical engineering project. Enterprise $\mathrm{H}$ is an engineering project contracting company, covering many fields such as electrical, civil engineering and construction, with a wide range of business. First, using the expert evaluation method, 20 project managers and enterprise risk management personnel are invited to evaluate and score various secondary indicators of $\mathrm{H}$ enterprise. According to the scores obtained from each indicator, use formula $r_{j m}=20 / n$ to obtain the degree of membership corresponding to each maturity level, $n$ is the number of experts corresponding to a certain maturity level under a single index. Then the secondary evaluation index matrix $R_{i}$ can be obtained.

(1) Take the second-level indicators $A_{11}$ and $A_{12}$ as examples. According to the expert scores, their secondlevel indicator evaluation matrix is:

$$
R_{1}=\left(\begin{array}{ccccc}
0 & 0.1 & 0.5 & 0.3 & 0.1 \\
0 & 0 & 0.7 & 0.25 & 0.05
\end{array}\right)
$$

Then use formula (4) to calculate the evaluation result matrix:

$$
\begin{aligned}
& B_{1}=W_{1} \circ R_{1}=\left[\begin{array}{ll}
0.6667 & 0.3333
\end{array}\right] \circ\left(\begin{array}{ccccc}
0 & 0.1 & 0.5 & 0.3 & 0.1 \\
0 & 0 & 0.7 & 0.25 & 0.05
\end{array}\right) \\
& =\left[\begin{array}{lllll}
0 & 0.0667 & 0.5667 & 0.2833 & 0.0833
\end{array}\right]
\end{aligned}
$$

In the same way, the matrix corresponding to the remaining secondary indicators can be calculated:

$$
\begin{aligned}
& B_{2}=\left[\begin{array}{lllll}
0 & 0.0989 & 0.4527 & 0.3921 & 0.0562
\end{array}\right] \\
& B_{3}=\left[\begin{array}{lllll}
0 & 0.2090 & 0.5397 & 0.2514 & 0
\end{array}\right] \\
& B_{4}=\left[\begin{array}{lllll}
0.0390 & 0.1627 & 0.6200 & 0.1783 & 0
\end{array}\right]
\end{aligned}
$$

Then according to formula (6), the maturity rating matrix can be calculated:

$$
A=W \times B_{i}=\left[\begin{array}{llll}
W_{1} & W_{2} & \ldots & W_{i}
\end{array}\right] \times\left(\begin{array}{c}
B_{1} \\
B_{2} \\
\ldots \\
B_{i}
\end{array}\right)
$$

$=\left[\begin{array}{llll}0.2359 & 0.5426 & 0.1221 & 0.0995\end{array}\right] \times\left(\begin{array}{ccccc}0 & 0.0667 & 0.5667 & 0.2833 & 0.0833 \\ 0 & 0.0989 & 0.4527 & 0.3921 & 0.0562 \\ 0 & 0.2090 & 0.5397 & 0.2514 & 0 \\ 0.0390 & 0.1627 & 0.6200 & 0.1783 & 0\end{array}\right)$

$=\left[\begin{array}{lllll}0.0039 & 0.1111 & 0.5069 & 0.3280 & 0.0501\end{array}\right]$

Then according to formula (5) and formula (7), the overall score of project risk management maturity and the score of various indicators are calculated:

$$
\begin{aligned}
& F=A \times V^{T}=\left[\begin{array}{llllll}
0.0039 & 0.1111 & 0.5069 & 0.3280 & 0.0501
\end{array}\right] \times\left[\begin{array}{lllll}
1 & 2 & 3 & 4 & 5
\end{array}\right]^{T} \\
& =3.3093 \\
& F_{1}=B_{1} \times V^{T}=\left[\begin{array}{lllll}
0 & 0.0667 & 0.5667 & 0.2833 & 0.0833
\end{array}\right] \times\left[\begin{array}{lllll}
1 & 2 & 3 & 4 & 5
\end{array}\right]^{T} \\
& =3.3832
\end{aligned}
$$

The same can be obtained:

$$
F_{2}=3.4053, F_{3}=3.0427, F_{4}=2.9376
$$

It can be seen that the risk management maturity score of $\mathrm{H}$ enterprise electrical engineering project is 3.3093, among which the senior management support index score is 3.3832, and Table 7 shows that its maturity level is standard; the overall capability index of the project risk management process is 3.4053, maturity level is standard; the corporate culture and organizational structure index score is 3.0427, and the maturity level is standard; the project risk management tools and methods application level index score is 2.9376 , and the maturity level is the managed level.

For $\mathrm{H}$ company, the overall ability of project risk management process and senior management support are relatively high scores, and it can continue to be lean to achieve the normative level; corporate culture and organizational structure basically achieve the standard level, and still need to continue Standardization; and the low score for the application level of project risk management tools and methods indicates that $\mathrm{H}$ enterprise still has insufficient application of risk management methods and tools and needs to further strengthen related management.

This empirical study is in line with reality and fully verified the scientificity and feasibility of the electrical engineering project risk management maturity model.

\section{References}

1. W.Chen, Research on Project Management Optimization of S Company Based on Maturity Model(MA thesis, Jilin University, 2019)

2. H.C.Jiang,M.S.Qiang,Pro.Man.Tec.07,17-22(2013)

3. J.C.Su, Research on Kangbashi Distribution Network Project Risk Management Based on Maturity Model(MA thesis, North China Electric Power University, 2016)

4. Zh.Q.Qin, Research on the Project Management Maturity Model Based on Grey Comprehensive Evaluation(MA thesis, Central South University, 2009)

5. K.Chen, Research on Application of Project 
Management Maturity Model in Construction Projects in China(MA thesis, Tongji University ,2006)

6. Y.Lu, H.Li, Pro.Man.Tec. 03, 38-41(2011)

7. J.X.Zhao, K.Fan, M.M.Wang, J.H.Sun, Jou.of.Eng.Man.01,134-139(2017):

8. M.Chen, Y.L.Zhao, Pro.Man.Tec.05,75-78(2017)

9. J.Guan, W.You, Pro.Man.Tec.05,62-70(2019) 